\title{
Norois
}

Environnement, aménagement, société

\section{Conservation des forêts et réorganisation spatiale de l'agriculture en pays betsileo (Madagascar) : essai de modélisation graphique}

Assessing spatial dynamics of agricultural activities in response to forest conservation policies (Betsileo country, Madagascar): graphic modeling as a method

\section{Aurélie Toillier}

\section{(2) OpenEdition}

\section{Journals}

Édition électronique

URL : https://journals.openedition.org/norois/2665

DOI : $10.4000 /$ norois. 2665

ISBN : 978-2-7535-1557-4

ISSN : $1760-8546$

\section{Éditeur}

Presses universitaires de Rennes

Édition imprimée

Date de publication : 15 décembre 2008

Pagination : 93-107

ISBN : 978-2-7535-0805-7

ISSN : 0029-182X

\section{Référence électronique}

Aurélie Toillier, «Conservation des forêts et réorganisation spatiale de l'agriculture en pays betsileo (Madagascar) : essai de modélisation graphique », Norois [En ligne], 209 | 2008/4, mis en ligne le 01 mars 2011, consulté le 14 janvier 2022. URL : http://journals.openedition.org/norois/2665 ; DOI : https://doi.org/10.4000/norois.2665 


\title{
CONSERVATION DES FORÊTS ET RÉORGANISATION SPATIALE \\ DE L’Agriculture EN PAYS BETSileo (MAdAgASCAR) \\ ESSAI DE MODÉLISATION GRAPHIQUE
}

\author{
AuréLIE ToIlLier \\ UMR METAFORT INRA/UR 199 IRD \\ (INRA/Agro Paris Tech - ENGREF), \\ Domaine des Cézeaux BP 90054, 24 Avenue des Landais - 63171 AubIÈrEs cedex 9 \\ (IRD), \\ BP 434 - ANTANANARIVO, Madagascar \\ aurelie.toillier@ird.fr
}

\section{RÉSUMÉ}

À Madagascar, depuis la fin des années 1990, des dispositifs de conservation des forêts appliqués à de petits territoires ruraux ont été mis en place dans le cadre de la décentralisation de la gestion des ressources naturelles. De nouvelles règles d'accès aux forêts et des zonages des territoires ont été définis. Les plans de zonage sont souvent élaborés sur des critères techniques, écologiques et touristiques, et correspondent rarement à des espaces sociaux et économiques réels. Les références explicites aux logiques agricoles sont quasiinexistantes. La gestion effective qui en découle peut alors présenter des effets pervers aussi bien du point de vue de la conservation que de celui du développement agricole. L'objectif de cette recherche est de formaliser une démarche pour comprendre et représenter la réorganisation des activités agricoles en réponse à ces nouvelles contraintes, afin qu'elle puisse être prise en compte dans des schémas d'aménagement du territoire conciliant conservation et développement. Pour analyser les logiques d'organisation aux deux niveaux, exploitation agricole et territoire, et leurs évolutions, puis pour les relier, la démarche proposée est basée sur l'utilisation conjointe de deux outils : un SIG et la modélisation graphique à l'aide de chorèmes. La nouvelle différenciation des espaces agricoles au sein du territoire ainsi que le rôle joué par les différents types d'organisation des exploitations ont pu être représentés. La modélisation graphique a contribué à l'identification d'enjeux d'aménagement du territoire induits par la conservation des forêts.

MOTS CLÉ : gestion contractualisée des forêts - conservation - organisation des activités agricoles - territoire - exploitation agricole - chorèmes - aménagement du territoire - Madagascar

\section{ABSTRACT}

Assessing spatial dynamics of agricultural activities in response to forest conservation policies (Betsileo country, Madagascar): graphic modeling as a method 
In Madagascar, forest conservation planning is based on a zoning system. It is and managed by local communities and takes place within small rural territories. The forest conservation assesment derives from environmental and ecological proxies and doesn't takes agriculture into account. Therefore the local farmers may have to face difficulties to cope with the new forest zoning system. This research aims at providing a better understanding of the reorganization of agricultural activities in order to improve their guidance. We tested an analytical approach based on a combination of GIS and choremes. New differentiations of agricultural patterns within the territory have been highlighted and the role played by different types of farms spatial behaviours has been represented. In conclusion graphic modelling allowed identifying land use planning issues induced by conservation measures.

KEY WORDS : community-based forest management-conservation-land-use - changes - land-use planning - farmland-graphic modeling - Madagascar.

Madagascar comme beaucoup de pays d'Afrique connaît des enjeux importants autour des modes de gestion et d'aménagement des petits territoires ruraux. Ceux-ci ont émergé dans le cadre de la décentralisation de la gestion des ressources naturelles, amorcée depuis une dizaine d'années pour enrayer la dégradation, voire la disparition, des écosystèmes en particulier forestiers (Batterbury, 1998). La reconnaissance des capacités de gestion des collectivités locales et de la nécessité pour l'État de déléguer l'autorité et la responsabilité de gestion des ressources, a conduit à la loi GELOSE ${ }^{1}$ promulguée en 1996 (Montagne et Ramamonjisoa, 2006). Elle institutionnalise le transfert de gestion des ressources naturelles aux communautés rurales et a été suivie en 2001 par un décret relatif à la gestion contractualisée des forêts de l'État $\left(\mathrm{GCF}^{2}\right)$ qui est spécifique aux ressources forestières. L'association des populations riveraines des forêts à la conservation est censée permettre une meilleure prise en compte de leurs besoins et ainsi assurer la pérennité des dispositifs (Froger et al., 2004). Les contrats de transfert de gestion sont issus d'une négociation entre le service des Eaux et Forêts (EEF), la communauté de base (COBA) et la commune sur les modalités d'accès et d'utilisation des produits forestiers et sur la délimitation des zones de conservation au sein d'un territoire villageois ou d'un fokontany ${ }^{3}$. Mais bien souvent, ces contrats ne prennent pas véritablement en compte les logiques d'occupation de l'espace par l'agriculture (Blanc-Pamard et Rakoto, 2007). En l'absence de compensations économiques satisfaisantes (Montagne et al., 2008), les paysans doivent envisager par eux-mêmes de nouvelles perspectives de développement dans les limites de leur territoire. La relative stabilité des lisières forestières et les changements paysagers dans les espaces cultivés, observés au sein des territoires villageois quelques années après la mise en place des GCF, nous font penser que les paysans peuvent changer leurs modes d'utilisation de l'espace pour s'adapter aux contraintes issues des mesures de conservation.

Afin de mieux prendre en compte les logiques agricoles dans la mise en ouvre de la GCF et de soutenir celles qui sont compatibles avec des objectifs de conservation et de développement, cette recherche propose une méthode d'analyse des processus de réorganisation des exploitations

1. La loi GELOSE 96-025 du 30 septembre 1996 est relative à la Gestion Locale Sécurisée des ressources naturelles renouvelables (RNR), en prenant compte de l'élément forêt, avec son décret d'application n² 2000-27 du 13 janvier 2000 relatif aux communautés de base chargées de la gestion locale des RNR.

2. La loi 97-017 du 08 août 1997 porte révision de la législation forestière avec son décret d'application n 2001-122 du 14 février 2001 fixant les conditions de mise en œuvre de la Gestion Contractualisée des Forêts de l'État (GCF).

3. Le fokontany constitue actuellement la plus petite unité administrative territoriale à Madagascar et peut être composé d'un ou plusieurs villages ou hameaux. Traditionnellement il correspond au territoire géré et utilisé par les membres d’un ou de plusieurs clans, descendants d'un même ancêtre. 
agricoles au sein de leur territoire. C'est au niveau de l'exploitation agricole que l'on peut cerner la transformation des activités de production et comprendre leur impact sur l'espace (Osty et al., 1998). C'est donc ce niveau que nous avons choisi de privilégier. Il existe cependant encore peu de méthodes opérantes pour décrire et comprendre le fonctionnement des exploitations agricoles dans leur territoire, en particulier en milieu rural africain (Gafsi et al., 2007).

Pour rendre compte des liens entre le fonctionnement des exploitations et leur inscription spatiale, plusieurs recherches se basent sur un ensemble de concepts et de méthodes couplant des outils de l'agronomie et de la géographie (Benoît et al., 2006). La modélisation graphique à l'aide des chorèmes en fait partie. Les chorèmes développés par R. Brunet (1980) donnent à voir de manière graphique les traits d'organisation spatiale et fonctionnelle des phénomènes étudiés par les géographes. Ce sont des formes symboliques représentant des structures ou des processus élémentaires dont l'assemblage dans un modèle graphique permet de représenter tout type de territoire (Brunet, 1986). La démarche repose sur une hypothèse de correspondance entre forme et fonctionnement (Lardon et al., 2005). Les changements d'organisation spatiale sont utilisés afin de révéler les processus fonctionnels dont ils sont à la fois facteurs et produits (Lardon et al., 2001 ; Bonin et Lardon, 2002).

À partir d'un cas d'étude dans la région du corridor forestier de Fianarantsoa (centre-est de Madagascar), il s'agit de montrer l'apport de cette démarche à la compréhension des changements induits par un dispositif de conservation par zonages sur l'organisation des activités agricoles.

\section{Contexte de la zone d'étude}

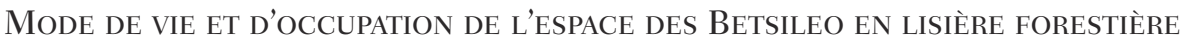

Dans la province de Fianarantsoa, au centre-est de Madagascar, les reliques de la forêt tropicale humide s'étendent de part et d'autre de la «falaise » longitudinale séparant la côte des Hautes-Terres. En lisière ouest, sur les hautes-terres, à une altitude moyenne de 1200 mètres, sont installés les Betsileos depuis plus d'un siècle. Dans un milieu vallonné, aux sols pauvres où la végétation herbacée domine, le système de production betsileo est basé sur l'association entre la riziculture de bas-fond, un petit élevage bovin servant aux travaux dans la rizière (piétinage, fumure), des cultures pluviales sur pente servant de substituts lors de la période de soudure (essentiellement manioc, taro et patates douces), et l'utilisation de produits forestiers. Il s'agit d'une agriculture d'autosubsistance. Les paysans se sont toujours servis de la forêt pour l'élevage (pâturage, reproduction et protection vis-à-vis des voleurs) et pour la collecte de produits forestiers (Moreau, 2002). Ces derniers sont très diversifiés et peuvent être destinés soit à des usages commerciaux, soit à leur autosubsistance. Bien souvent ils constituent un revenu non négligeable lors de la période de soudure. Il s'agit de produits non ligneux comme le miel, les écrevisses, les fruits, les plantes médicinales, des matières premières pour l'artisanat, l'habillement, ou la construction des habitations (feuilles, bambous). Le bois est utilisé pour la fabrication d'outils agricoles, des clôtures, la charpente des cases, pour le bois de chauffe.

Le caractère accusé du relief, l’hétérogénéité du milieu qui s'ensuit et l'association de l'agriculture et de l'élevage ont conduit les paysans betsileo à organiser spatialement leur production en jouant sur cinq facettes paysagères (Blanc-Pamard et Milleville, 1991) : les farihy (rizières de bas-fond); les kipahy (rizières de versant en terrasses encore rares); les tambina (cultures vivrières de complément installées sur de petites parcelles de bas de pente); les vodivala (vergers d'arbres fruitiers, bananiers, canne à sucre, localisés en aval des parcs à bœufs); les tamboho (sommets de colline réservés au pâturage en période humide). Des bosquets d'eucalyptus marquent aussi sur les crêtes, des limites de domaines fonciers familiaux. En forêt, seuls les deux tiers inférieurs des versants sont généralement cultivés, selon une ancienne réglementation imposée par le service des Eaux et Forêts pour limiter l'érosion. 


\section{LES CONTRAINTES ISSUES DU DISPOSITIF DE CONSERVATION}

Le territoire d'étude retenu est le fokontany d'Iambara, situé dans la zone périphérique du parc national de Ranomafana (fig. 1). La déforestation est essentiellement due à un recul lent de la lisère sous l'action des feux de brousse servant à l'entretien des pâturages, à des défriches autour de bas-fonds pour l'aménagement en rizière, et à des défriches occasionnelles pour une culture d'appoint (maïs, haricot) lorsque les productions vivrières sont insuffisantes.

Certains villages sont installés au cœur de la forêt depuis le début du XXe siècle : ils sont liés à d'anciennes activités coloniales (or, bois précieux, construction des voies de communication). Les défrichements ont essentiellement pour but la mise en place de terroirs agricoles pérennes autour de bas-fonds aménagés en rizière avec un habitat fixe.

Un dispositif de GCF a été mis en place en 2002 (fig. 2a). Il consiste en un zonage des 1492 hectares de massifs forestiers utilisés par l'ensemble des 13 villages installés dans, et en lisière de la forêt. Il est constitué d'une zone de protection intégrale dans sa partie nord et d'une zone de conservation dans sa partie sud, au sein de laquelle une zone de droits d'usage de 45 hectares est autorisée pour la collecte de bois, uniquement à des fins non commerciales. Sa localisation, qui doit changer tous les trois ans, est en partie choisie par les villageois en fonction des ressources utilisables et de son accessibilité. Les feux de brousse pour le renouvellement des pâturages en savane ont été interdits et la constitution de pare-feux est obligatoire autour des jachères défrichées avant le brûlis. Les jachères présentant des arbustes de diamètres supérieurs à $30 \mathrm{~cm}(\mathrm{dbh})$, ne doivent plus être défrichées.
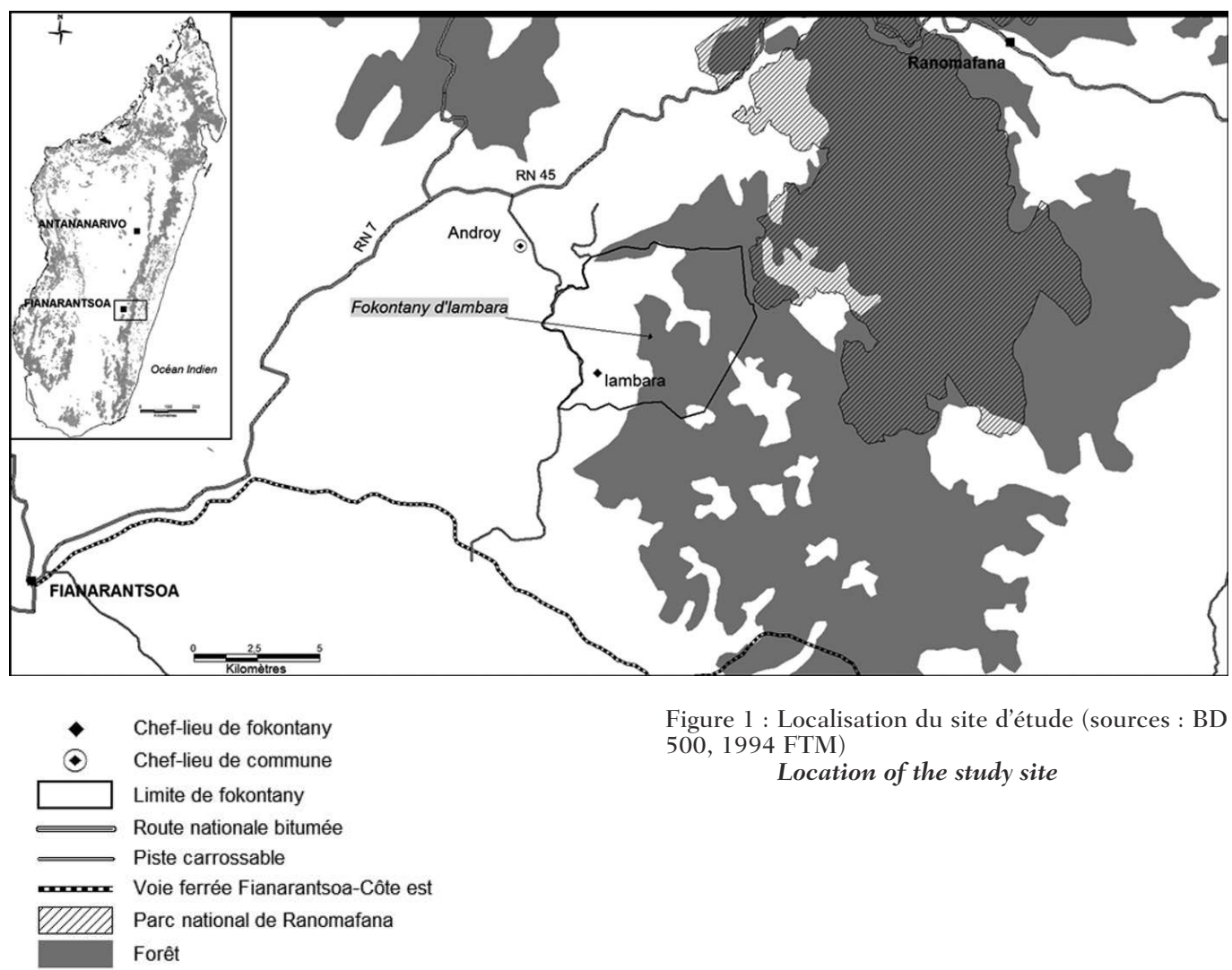

Figure 1 : Localisation du site d'étude (sources : BD 500, 1994 FTM)

Location of the study site 
Le service des Eaux et Forêts a accepté de considérer le besoin en rizières des villageois en autorisant la défriche de forêt de part et d'autre des bas-fonds en cours d'aménagement dans une limite maximale de 25 mètres sur les versants, afin de favoriser l'ensoleillement de la rizière.
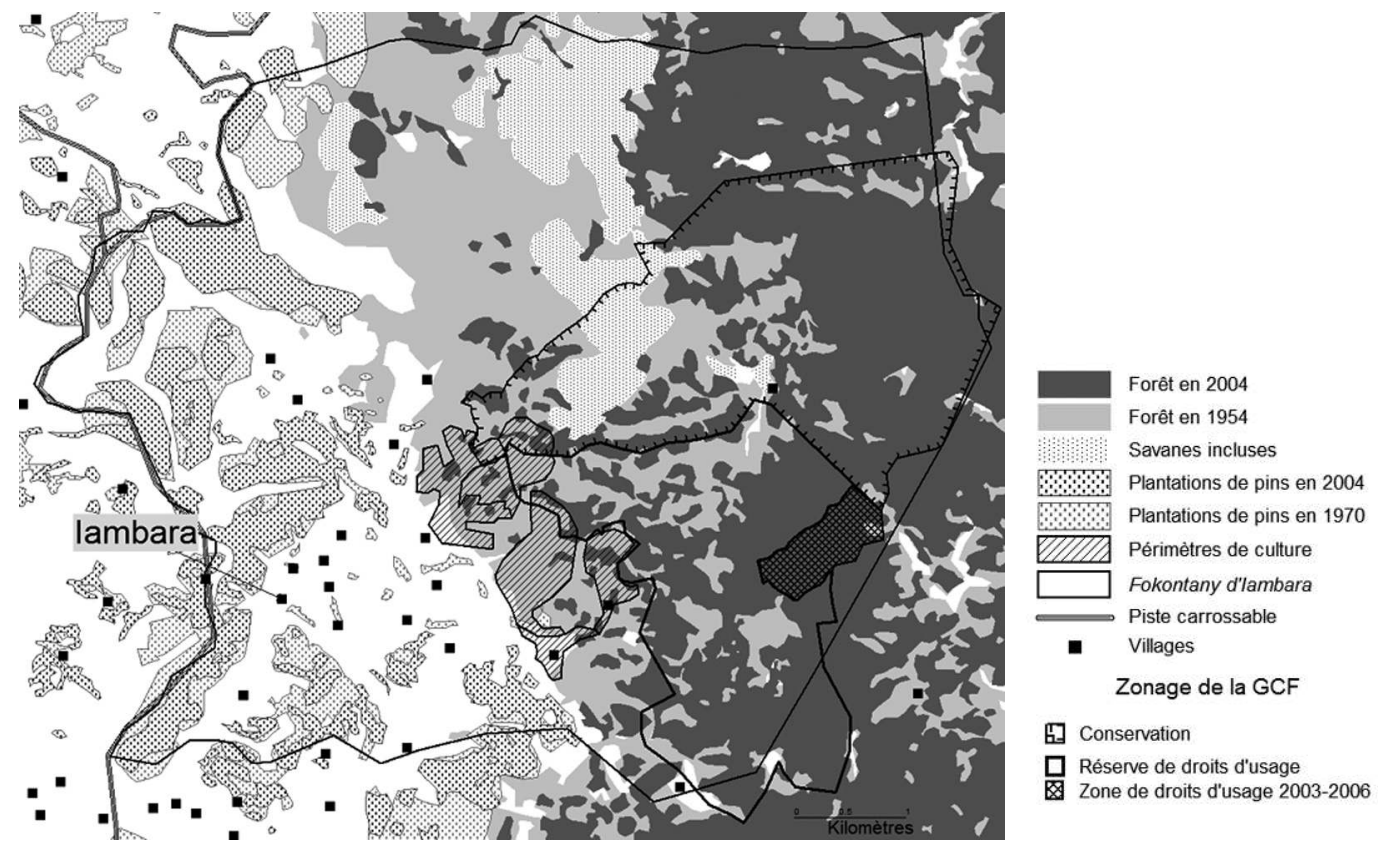

Figure 2a : Évolution des surfaces boisées entre 1957 et 2004, localisation des villages, périmètres de cultures accordés par le service des Eaux et Forêts et zonages de conservation (GCF)

GCF zoning plan and land cover evolution in the study site (Fokontany of Iambara) between 1954 and 2004: forest and pine plantations (sources: interpretation of aerial photographs 1954, forest service maps 1970BD 500 FTM 1994 and SPOT image 10m., 2004).

\section{Démarche d'analyse des changements d'organisation spatiale des activités agricoles en réponse au dispositif de conservation}

Nous cherchons à rendre compte d'un processus à forte dimension spatiale : la réorganisation des activités agricoles en réponse aux contraintes d'un dispositif de conservation territorialisé des forêts. Nous avons donc choisi l'espace comme entrée privilégiée des analyses, en la couplant à une analyse fonctionnelle des exploitations agricoles, unité de base de prise de décision et de gestion de l'espace. Pour introduire la dimension spatiale dans une typologie de fonctionnement des exploitations, nous avons au préalable procédé à un découpage de l'espace.

\section{DÉCOUPAGE DE L’ESPACE POUR RELIER FONCTIONNEMENT DES EXPLOITATIONS ET ORGANISATION SPATIALE DES ACTIVITÉS AGRICOLES}

Pour relier des processus au niveau des exploitations, à des entités spatiales plus englobantes significatives pour les enjeux environnementaux, nous avons choisi de nous appuyer sur la méthode des unités agro-physionomiques (UAP) définies par P. Thinon et J.-P. Deffontaines (1999). Ce sont des unités spatiales intermédiaires de relative égale apparence selon des critères écologiques et agronomiques au sein desquelles les ressources, leur utilisation, leur mise en valeur et les dif- 
AurÉlie TOILliER

ficultés rencontrées constituent une problématique « homogène » dont la variabilité est minime à l'échelle retenue (Bonin et al., 2001).

Les méthodes de construction reposent sur l'enchainement de plusieurs phases, alternant des approches au niveau de l'ensemble de la zone d'étude et des approches locales. Elles comprennent un va-et-vient entre des études de terrain (analyses paysagères, entretiens) et des analyses de documents cartographiques et photographiques (Deffontaines, 2006).

Des sous-zones dites « homogènes » ont été identifiées par reconnaissance sur le terrain, analyse spatiale de photographies aériennes et cartes ${ }^{4}$ intégrées dans un Système d'Information Géographique (SIG). Ont été extraits : i) une représentation des caractéristiques du milieu qui conditionnent les installations agricoles (réseau hydrographique, relief, sol); ii) la localisation des zones de culture légales (statut foncier, périmètres de culture accordés par les Eaux et Forêts); iii) la localisation des villages, des pistes; iv) l'évolution des principaux types d'occupation du sol (reboisements de pins, forêt, jachères arborées ou herbacées, cultures, prairies et rizières) (fig. 2a).

Le croisement des sous-zones avec le territoire des exploitations permet d'introduire la répartition des sous-zones comme critère de typologie de ces dernières (Deffontaines, 1996). Ainsi en reliant une typologie de l'espace rural (sous forme de zonages) à une typologie spatialisée du fonctionnement des exploitations agricoles, il sera possible d'une part de prévoir les effets sur le territoire d'une modification des systèmes de culture et, d'autre part, de prévoir les conséquences sur les divers systèmes de culture d'une transformation du territoire, telle que celle liée au dispositif de conservation.

\section{ÉLABORATION D’UNE TYPOLOGIE SPATIALISÉE DES EXPLOITATIONS}

Pour analyser les changements d'organisation suite à la mise en place de la GCF, nous avons choisi d'élaborer une typologie spatialisée des exploitations qui caractérise leur fonctionnement avant la mise en œuvre du dispositif de conservation. Les stratégies d'adaptation à la conservation et les changements d'organisation des territoires d'exploitation qui en ont découlé, sont ensuite analysés pour chaque type d'exploitation et représentés par des chorèmes.

Un échantillon de 32 exploitations réparties au sein des sous-zones a été défini. Les enquêtes réalisées en 2006, soit 4 ans après la mise en ouvre de la GCF, sont constituées de 3 parties. La première partie est fondée sur des questions fermées pour caractériser le ménage, ses systèmes de production et d'activités, ses objectifs de production et de reproduction. La deuxième partie est focalisée sur la structure actuelle du territoire d'exploitation, les étapes de sa constitution et de son utilisation, et les raisons de chaque changement évoqués afin de repérer les changements opérés en lien avec les contraintes du dispositif de conservation. Une cartographie du territoire est réalisée à l'aide d'un GPS ${ }^{5}$. La dernière partie est un entretien ouvert sur les perceptions du ménage des avantages et inconvénients de la GCF et des impacts sur leurs activités et mode de vie.

Sur la base de 16 variables décrivant la stratégie d'utilisation des ressources et de 6 variables décrivant l'organisation spatiale des exploitations, une typologie spatialisée a été constituée et validée sur un échantillon plus large (Toillier et al., 2009). Les variables structurelles sont liées aux capitaux (force de travail, animaux et matériel, superficies de rizière), aux objectifs de production et sources de revenu. Les variables spatialisées (répartition des champs dans les sous-zones, distance à la résidence principale, mode d'occupation du sol) sont analysées et représentées à l'aide d'un SIG et de modèles graphiques.

Les modes d'organisation spatiale des exploitations ont été décrits en fonction de la répartition des champs entre les sous-zones et de leur utilisation. Les traits de structure élémentaires pour représenter les modes d'organisation des exploitations sont : les cases d'habitation principale et secondaire, les parcs à bœufs, l'usage des champs sur crêtes, versants, thalwegs dans les têtes de

4. Cartes topographiques FTM 1976, photographies aériennes (1957 et 1991, FTM) et de l'image SPOT5 2004 à 10 m de résolution (copyright CNES-ISIS). FTM : Foiben-taosarintanin’i Madagasikara, institut géographique de Madagascar.

5. Global Positionning System. 
vallon et bas-fonds. Pour les champs sur versants, sont distinguées les cultures pérennes des cultures annuelles et pour les jachères, sont identifiées les jachères arborées (qui après un certain stade peuvent potentiellement être incluses dans la GCF), les jachères herbacées dont le retour à un état forestier est impossible et qui marquent un épuisement du sol (Randriamalala et al., 2007).

Des chorèmes ont été construits pour représenter l'organisation spatiale des différents types d'exploitation. Pour cela les représentations cartographiques des territoires et de leur utilisation sous le SIG, ont aidé à identifier des formes d'organisation récurrentes, les structures élémentaires et leurs différentes combinaisons. Nous avons retenu des modèles graphiques en nous appuyant également sur des travaux antérieurs portant sur le même type de problématique (Albaladejo et Lardon, 1990 ; Bonin, 2002). Puis, pour chaque type, nous avons identifié les différentes combinaisons de changement de mode d'organisation effectué après conservation. Elles ont été analysées en regard des logiques de production et d'utilisation des ressources naturelles, avant et après la mise en œuvre du dispositif de conservation.

\section{Résultats}

L'identification de sous-zones agro-écologiques homogènes, dans le territoire du fokontany d'Iambara, où les options d'usage du sol sont les mêmes pour l'ensemble des ménages, définit en partie les modes d'organisation spatiale des exploitations. Cinq types spatialisés d'exploitation ont été identifiés. Leurs stratégies d'adaptation à la conservation a conduit à de nouveaux modes de mise en valeur de leur territoire, qui permettent d'expliquer, au sein des sous-zones, les grandes tendances d'évolution paysagère.

\section{Trois SOUS-ZONES AGRO-ÉCOLOGIQUES HOMOGÈNES}

Trois zones agro-écologiques homogènes ont été identifiées au sein du territoire du fokontany (fig. 2b).

- La zone dite de «savane » (zone 1) correspond aux « vieux » terroirs où les villages sont installés depuis plus d'un siècle près de grands bas-fonds aménagés en rizières. La riziculture est associée à l'élevage de zébus. Les versants sont utilisés pour les cultures pluviales (manioc, patate douce, taro, pois de terre, arachide) cultivées en association, et en alternance parfois au cours de nombreux cycles successifs, avec de très courtes périodes de mise en jachère ( 1 à 2 ans). Le sommet des collines est réservé aux pâturages ou aux plantations d'eucalyptus à usage privé. De grandes superficies ont été récupérées par l'État dans les années 1960 pour la plantation de pins et par des usines de tanins pour la plantation d'acacias.

- La zone 2 correspond à la lisière forestière qui a toujours été utilisée par les habitants des villages installés dans la zone de « savane ». Elle comprend les périmètres de culture accordés par le service des Eaux et Forêts dans les années 1970 et 1990, en contrepartie des terres boisées en pins qui avaient été récupérées. Il s'agit de têtes de vallons entièrement couvertes de forêt naturelle, qui ont été divisées entre les habitants demandeurs de terres dans les différents villages de lisière. Les cultures de maïs et haricot y sont pratiquées avec des jachères longues (4 à 10 ans) et dans certains cas une pérennisation de l'occupation agricole avec plantation de bananiers et de canne à sucre pour la fabrication de rhum local.

- Enfin la zone 3 correspond à une zone forestière dont la géomorphologie diffère. Ici, les bas-fonds sont peu nombreux, étroits et tourbeux donc difficiles à aménager pour la riziculture. Son éloignement des centres villageois en fait une zone difficile d'accès, au sein de laquelle tout aménagement agricole demande une installation permanente pour surveiller les cultures.

La figure $2 \mathrm{~b}$ représente les trois sous-zones identifiées, ainsi que les principales structures spatiales : relief, cours d'eau, zones d'habitats et axes de communication. 


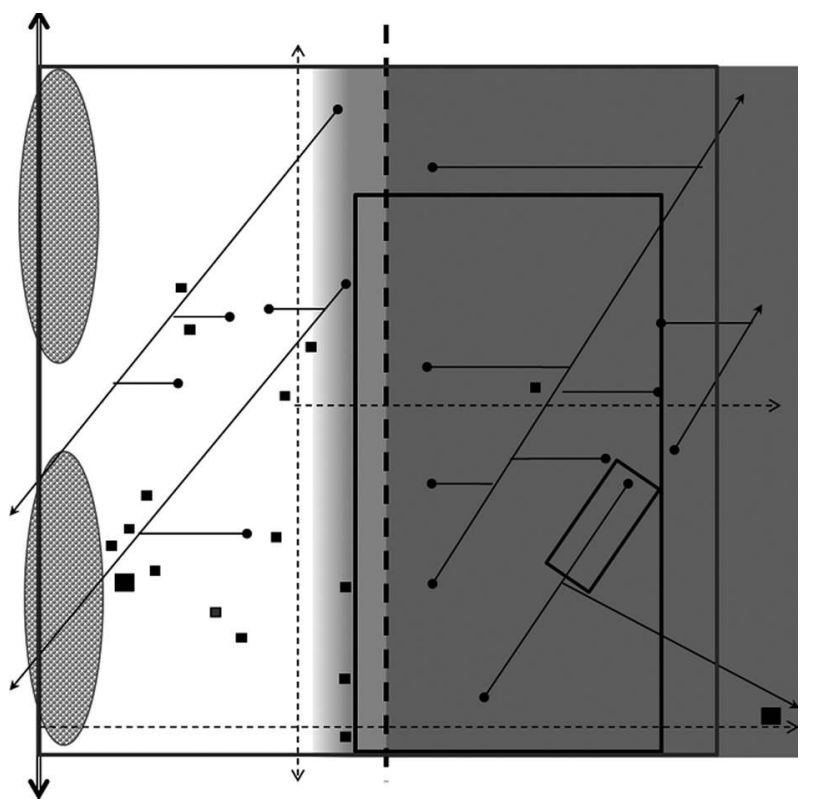

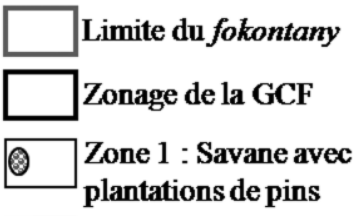

Zone 2 : Lisière forestière

Zone 3 : Forêt

$\longleftrightarrow$ Source et cours d'eau

- - Ligne de partage des eaux

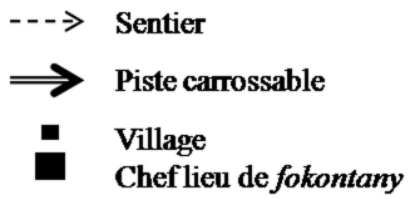

Figure 2b : Découpage du territoire du fokontany d'Iambara en sous-zones homogènes d'usage du sol et principaux traits de structure.

Graphic model of land use patters at territory scale (fokontany of Iambara): identification of three zones where settlement history and land-use options for farmers were similar

\section{STRATÉGIES D'ADAPTATION À LA CONSERVATION \\ ET CHANGEMENT D’ORGANISATION DES TERRITOIRES D'EXPLOITATION}

Cinq types d'organisation spatiale reliée à un mode de fonctionnement des exploitations agricoles ont été identifiés et représentés par des chorèmes (fig. 3).

À chaque type d'exploitation a correspondu un ensemble de stratégies, en réponse aux différentes contraintes issues des mesures de conservation. Les chorèmes ont été reconstruits pour chaque type en fonction des principales dynamiques de réorganisation des activités (fig. 4).

Le type 1 est composé d'exploitations installées en savane, contraintes par une petite superficie, un parcellaire très morcelé et des terres peu productives. Leur objectif est la recherche de revenus quotidiens rapides. La vente de produits forestiers (manches d'outils, écrevisses, paniers en bambou) ou la pratique de défriches isolées en forêt pour cultiver du haricot, leur permettaient de compléter les manques de production, en particulier lors de la période de soudure qui dure près de 6 mois. Leur mode d'organisation spatiale est qualifié de "morcelé en savane ». Leur stratégie d'adaptation a correspondu à une extension des champs de versant, en particulier sur les plantations de pins et d'acacias actuellement abandonnées par leurs gestionnaires. Il s'agissait pour eux d'un retour légitime sur leurs terres. Cette extension s'est accompagnée d'une intensification des cultures avec labour, raccourcissement des jachères, aménagement de terrasses anti-érosives ainsi qu'un aménagement pérenne des jachères ligneuses dans la zone 2 avec plantations de cannes à sucre et aménagement de rizières en terrasses dans les thalwegs et têtes de vallon. La production de rhum local a remplacé la vente des produits forestiers.

Le type 2 appelé « dédoublé », est composé des exploitations les plus capitalisées, mettant en œuvre une stratégie de conquête foncière des bas-fonds localisés en forêt. Une forte force de 


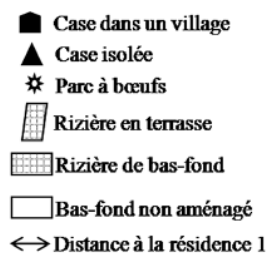

Figure 3 : Modèles graphiques des types d'exploitations et de leur mode d'organisation avant la mise en œuvre du dispositif de conservation (GCF)

Type 1: morcelé en savane, type 2 : Dédoublé savaneforêt, type 3 : divisé en transition, type 4 : dispersé en forêt, type 5 : groupé.

Graphic models of land use pattern preGCF of each household type b) post-GCF

Type 1: Divided savannah farm, type 2: Scattered mixed farm, type 3: Split mixed farm, type 4: Scattered forest farm, type 5: grouped farm.

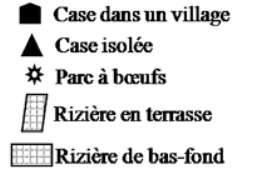

Bas-fond non aménagé

$\longleftrightarrow$ Distance à la résidence 1

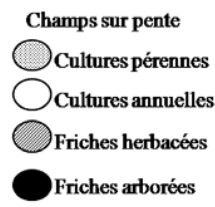

Friches arborées
Figure 4 : Modèles graphiques des types d'exploitations et de leur mode d'organisation après la mise en œuvre du dispositif de conservation (GCF)

Type 1 : morcelé en savane, type 2 : Dédoublé savaneforêt, type 3 : divisé en transition, type 4 : dispersé en forêt, type 5 : groupé.

Graphic models of land use pattern postGCF of each household type

Type 1: Divided savannah farm, type 2: Scattered mixed farm, type 3: Split mixed farm, type 4: Scattered forest farm, type 5: grouped farm
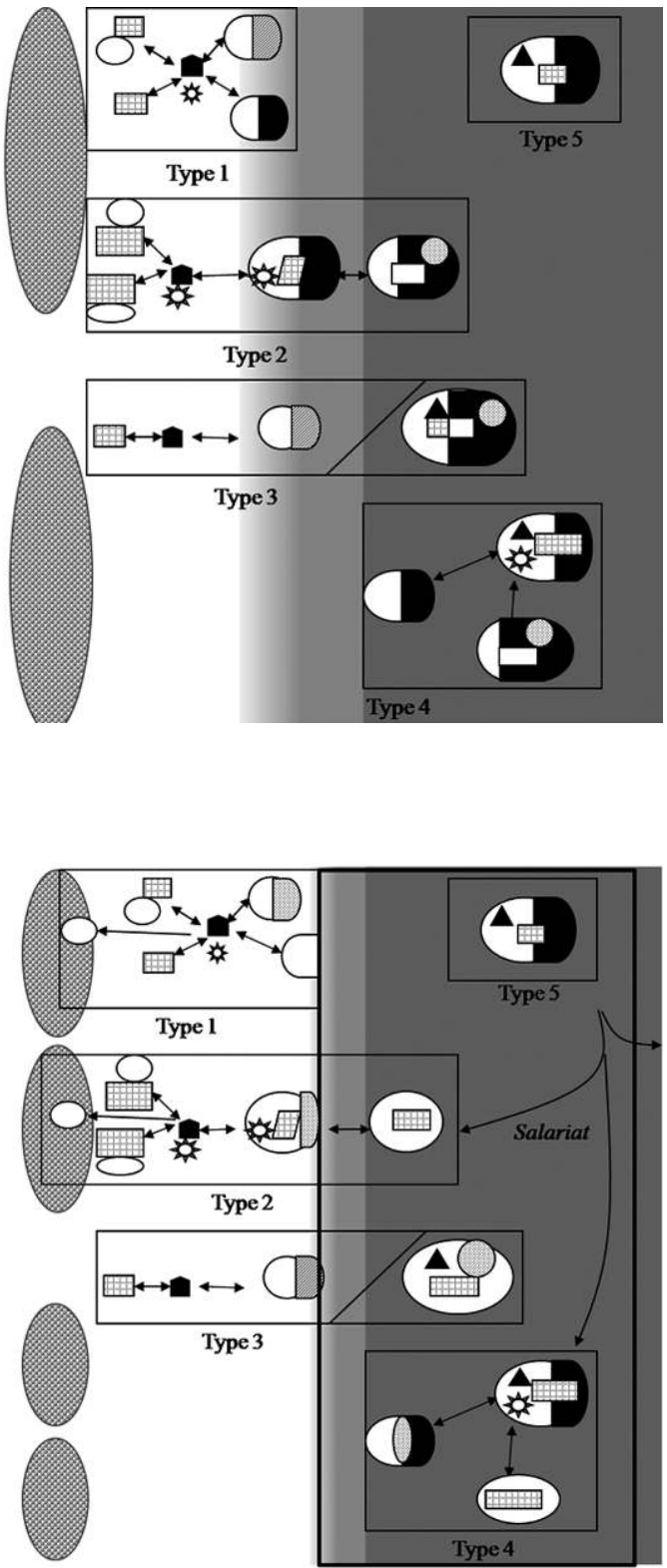

travail complété par de l'entraide familiale et l'embauche de salariés leur permettent d'intensifier et diversifier les cultures.

Les exploitants de type 3 partagent leur résidence entre un village en savane (zone 1) et une case isolée en forêt (zone 3), où ils ont récemment aménagé des rizières. Ce sont des ménages en phase d'installation avec très peu de terres héritées en savane. Ils développent des cultures de rente peu exigeantes en main-d'œuvre (canne à sucre) en bordure de rizières en forêt et vendent des produits forestiers pour compléter leur revenu. Leur objectif est d'aménager un nouveau domaine. C'est une organisation spatiale « divisée » qui traduit une étape de transition entre deux modes de fonctionnement. 
Les exploitations des types 2 et 3 ont profité de la règle de défriche de 25 mètres autour des bas-fonds pour aménager davantage de rizières en forêt (zone 3). Pour compenser les pertes de revenus issus de la vente des produits forestiers, les jeunes (type 3) ont développé de nouvelles sources de revenu, basées sur l'élevage de porcs organisé collectivement au village, et gardent ainsi le système de double résidence.

Les types 4 et 5 sont localisés en forêt.

Le type 4 (« dispersé en forêt ») est l'équivalent du type 2 mais est composé de ménages originaires des villages de forêt. Les exploitations sont organisées autour d'un ou plusieurs bas-fonds (zone 3) et pratique une agriculture semi-itinérante sur abattis-brûlis autour des bas-fonds. Pour pallier l'arrêt de la défriche de jachères arborées ou de nouvelles zones forestières, les exploitations du type 4 ont utilisé les temps morts du calendrier agricole, pour aménager davantage leurs champs présentant des sols moins fertiles et laissés auparavant en jachère. Ils y ont installé des cultures de manioc et de patates douces. Grâce à l'entraide familiale, une stratégie d'aménagement des petits bas-fonds tourbeux et contraignants, a également pu être initiée.

Le type 5 ("groupé ») est composé d'exploitations de petite superficie, groupées autour d'un bas-fond aménagé en rizière. Il s'agit soit de jeunes migrants, sans terres en savane, soit de retraités qui ont cédé leurs terres dans les vieux terroirs villageois en savane à leurs descendants, afin que les jeunes restent au village et bénéficient de la vie communautaire (entraide pour les travaux des champs, partage de capitaux comme des zébus avec d'autres jeunes). Le salariat et la culture de canne à sucre pour la fabrication de rhum sont les principales sources de revenu; les productions vivrières sont très insuffisantes et la période de soudure dépasse les huit mois. Les exploitants ont principalement allongé leur période de salariat en dehors de leur exploitation et augmenté les superficies plantées en canne à sucre. La diminution des surfaces de cultures vivrières et du travail réalisé sur l'exploitation, a provoqué une diminution des rations alimentaires

\section{Grandes TENDANCES D’ÉVOLUTION PAYSAGÈRE AU SEIN DES SOUS-ZONES}

En réponse aux contraintes de la conservation, certains types d'exploitation ont mis en œuvre de nouvelles pratiques agricoles et de nouveaux aménagements du milieu, qui se traduisent par des évolutions paysagères : extensions ou rétractions de l'espace cultivé; changements de types de culture; changements de répartition spatiale des cultures et des activités (tab. 1). Les extensions sont dues à l'aménagement des bas-fonds en rizière et à la mise en culture de terres forestières ou de prairies. Des changements dans la hiérarchie des cultures, l'intensification ou l'augmentation du taux de terres cultivées chaque année sont considérés comme une stabilisation de l'aire cultivée. La diminution de l'aire cultivée coïncide avec l'abandon des jachères herbacées devenues impropres à la culture par manque de fertilité, ou l'abandon de jachères arborées suite à leur insertion dans le zonage de conservation de la GCF.

Le tableau 1 met en relation les dynamiques paysagères observées au sein des sous-zones, avec les changements d'usage du sol mis en œuvre par les exploitations qui possèdent des champs dans la souszone considérée. Par exemple, vingt et une des exploitations enquêtées possèdent des champs en zone de savane et $19 \%$ d'entre elles ont étendu leur surface cultivée, uniquement sur les versants.

Paradoxalement les adaptations qui ont conduit à une extension de l'aire cultivée ont eu lieu dans la forêt (zone 3, $44 \%$, tableau 1). Elle est due à l'aménagement en rizières des nombreux bas-fonds dispersés en forêt. Cet aménagement a été systématiquement accompagné de la défriche de la forêt dans les 25 mètres autorisés de part et d'autre du bas-fond, quelle que soit l'inclinaison du versant. Généralement le ménage construit sa case à mi-pente afin de surveiller ses cultures, mais surtout pour montrer l'appropriation des lieux. L'aménagement intensif de rizières suivant les cours d'eau d'aval en amont s'est accéléré. Autrefois les ménages partageaient leur résidence entre le village et la forêt. Aujourd'hui des habitats permanents le long des bas-fonds apparaissent. Ces nouveaux lieux d'habitation sont dispersés en forêt et isolés au milieu de petites clairières agricoles. Cette nouvelle 


\begin{tabular}{|c|c|c|c|c|c|c|}
\hline Sous-zone d'usage homogène du sol & \multicolumn{2}{|c|}{ Savane (1) } & \multicolumn{2}{|c|}{ Lisière ( 2) } & \multicolumn{2}{|c|}{ Forêt (3) } \\
\hline $\begin{array}{l}\text { Nb. d'exploitations possédant des champs dans } \\
\text { chacune des zones }\end{array}$ & 21 & & 29 & & 27 & \\
\hline $\begin{array}{l}\text { Extension de l'aire cultivée } \\
\text { Dont sur: } \\
\text { - Rizières et versants } \\
\text { - versants }\end{array}$ & $\begin{array}{l}19 \% \\
- \\
-\end{array}$ & $\begin{array}{l} \\
0 \% \\
100 \%\end{array}$ & $\begin{array}{l}34 \% \\
- \\
-\end{array}$ & $\begin{array}{l} \\
50 \% \\
50 \%\end{array}$ & $\begin{array}{l}44 \% \\
- \\
-\end{array}$ & $\begin{array}{l} \\
100 \% \\
0 \%\end{array}$ \\
\hline $\begin{array}{l}\text { Stabilité de l'aire cultivée } \\
\text { Dont: } \\
\text { - Augmentation du taux de terres cultivées chaque } \\
\text { année } \\
\text { - intensification } \\
\text { - Augmentation des cultures de rente (canne à } \\
\text { sucre) } \\
\text { - Changements dans la hiérarchie des cultures } \\
\text { - Pas de changements }\end{array}$ & $\begin{array}{l}- \\
- \\
- \\
- \\
-\end{array}$ & $\begin{array}{l}0 \% \\
73 \% \\
0 \% \\
20 \% \\
27 \%\end{array}$ & $\begin{array}{l}- \\
- \\
- \\
- \\
-\end{array}$ & $\begin{array}{l}29 \% \\
0 \% \\
36 \% \\
14 \% \\
29 \%\end{array}$ & $\begin{array}{l}- \\
- \\
- \\
- \\
-\end{array}$ & $\begin{array}{l}33 \% \\
17 \% \\
33 \% \\
25 \% \\
25 \%\end{array}$ \\
\hline Diminution de l'aire cultivée: & $10 \%$ & & $18 \%$ & & $11 \%$ & \\
\hline Total & $100 \%$ & & $100 \%$ & & $100 \%$ & \\
\hline
\end{tabular}

Tableau 1 : Changement d'usage du sol à l'échelle du territoire : stabilité, augmentation, ou diminution de la surface cultivée dans chaque zone, en \% du nombre d'exploitations

Land use changes in each land-use zone in function of patterns in cultivated area at the farm level (extension, decrease or stability)

configuration augmente les zones de contact entre forêt et agriculture, et aggrave les conditions de vie des ménages (déprédation aux champs et non maîtrise des techniques de piégeage). Ils souffrent également de nombreuses maladies, liées au froid et à l'humidité qui prévalent en forêt.

Dans les zones 1 et 2 , les principales stratégies d'adaptation à la conservation ont correspondu à une stabilité de l'aire cultivée, liée à une intensification pour les cultures vivrières dans la première zone et à la plantation de cultures de rente et pérennes (canne à sucre, bananes). Dans ces zones, des extensions sont observées dans quelques cas ( $19 \%$ en savane et $34 \%$ en lisière) et ont eu principalement lieu sur des zones boisées. Les plantations de pins ont été converties en champs de manioc. Les jachères arbustives en lisière ont presque toutes été coupées, afin d'éviter leur inclusion dans le zonage de conservation qui peut se produire quand les arbres atteignent 30 centimètres de diamètre, limite au-dessus de laquelle les arbres ne doivent plus être coupés. Les paysages de la savane et de lisière apparaissent aujourd'hui moins densément boisés. La lisière forestière est plus nette, suivant les contours de la GCF. Auparavant la mosaïque de jachères de différents âges et de champs cultivés, ne permettait pas de distinguer nettement la limite entre la forêt et les terroirs agricoles.

La diminution de l'espace cultivé s'est produite essentiellement en lisière et résulte de l'abandon de jachères herbacées. L'interdiction des feux annuels pour maintenir les pâturages a provoqué l'envahissement par des arbustes et buissons des prairies proches de la lisière forestière. D'après les paysans, tout feu accidentel durant une saison sèche risquerait de se propager très rapidement et serait difficile à arrêter.

La localisation dans le territoire des modes d'organisation des exploitations en réponse à la GCF, a permis d'identifier les dynamiques affectant chacune des zones et de faire apparaitre ainsi une nouvelle organisation des espaces agricoles au sein du territoire (fig. 5). Certaines dynamiques préexistantes ont été renforcées, comme l'accélération du « mitage » de la forêt, aujourd’hui localisé le long des cours d'eau par aménagement des bas-fonds en rizières. De nouvelles dynamiques d'occupation de l'espace sont apparues avec une valorisation des terres de collines en savane par extension et intensification des cultures; un embroussaillement des pâturages; une diminution des jachères en 
lisière et en forêt remplacées par de l'agroforesterie. Ces dynamiques ont abouti à une reconfiguration des zones de contact forêt-agriculture avec une évolution vers un paysage plus cultivé.

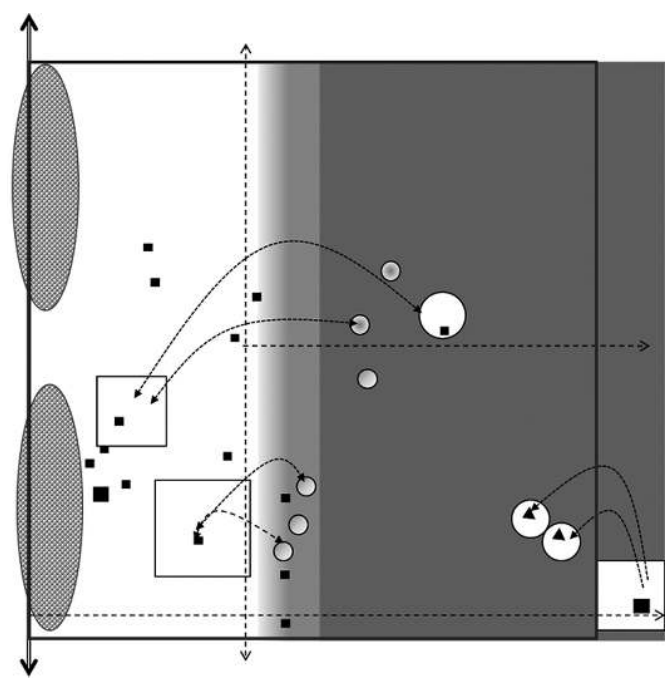

a)

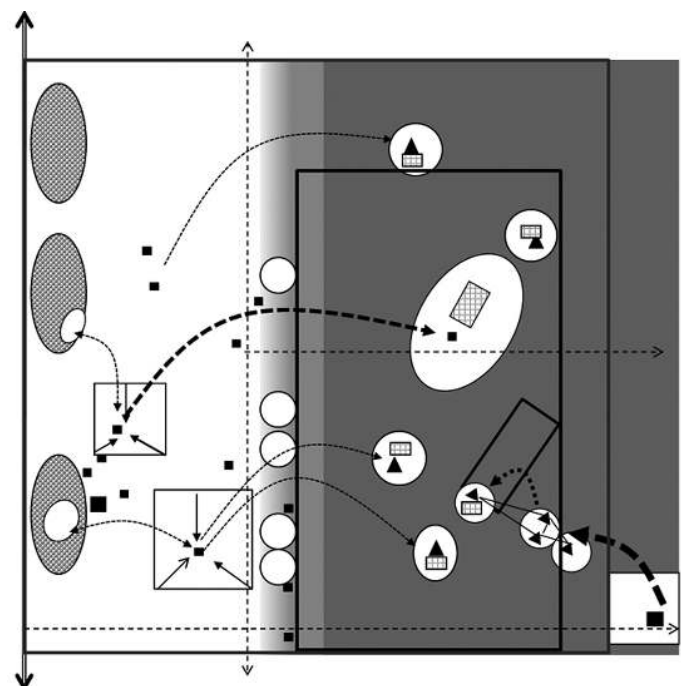

b)

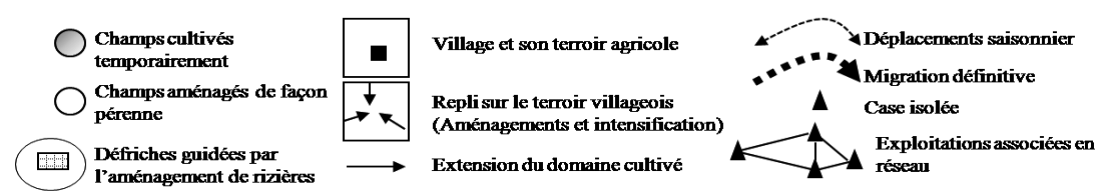

Figure 5 : Traits de dynamique spatiale des activités agricoles au niveau du territoire a) avant et b) après la GCF Graphic models of agricultural dynamics a) pre-and b) post-conservation at the territory level

\section{Discussion et conclusion}

\section{LES APPORTS DE LA DÉMARCHE DE MODÉLISATION GRAPHIQUE}

Nous avons cherché à rendre compte de la réorganisation des activités agricoles en réponse à un dispositif de conservation mis en œuvre dans un fokontany, à partir du fonctionnement des exploitations agricoles.

La démarche de modélisation graphique élaborée est basée sur l'utilisation des résultats de deux typologies d'espace : l'une au niveau du territoire du fokontany, au regard de ses caractéristiques agro-écologiques et foncières, et l'autre au niveau des exploitations agricoles au regard de leur mode de fonctionnement. Nous en avons dégagé des structures et dynamiques spatiales, aux différents niveaux, qui nous ont semblé rendre compte au mieux des processus de réorganisation des activités agricoles, en réponse aux contraintes du dispositif de conservation. La modélisation graphique présente l'avantage de pouvoir également prendre en compte des éléments sans empreintes spatiales comme les relations entre exploitations, les flux de population ou la polarisation des dynamiques agricoles (zones d'attraction, de pression). L'insertion des exploitations dans un ensemble territorial plus englobant est en effet indispensable à leur compréhension. Les exploitations établissent de nombreuses relations entre elles (techniques, économiques, familiales...) qui permettent de comprendre les choix techniques, les pratiques, et pour certains types, 
les stratégies d'adaptation. C. Blanc-Pamard et H. Rakoto (2007) montrent que l'accès facilité aux bas-fonds de certains exploitants se justifie au niveau des organisations lignagères. Ces aspects thématiques ont été peu développés dans cet article, car nous avons avant tout souhaité mettre en évidence la diversité du fonctionnement et des stratégies des exploitations, jusqu'à présent peu formalisés sur le plan méthodologique.

La standardisation que représente la mise en chorème permet, par ses qualités de synthèse cognitive, de comparer plus aisément des situations d'exploitations agricoles et leurs changements. Pour passer d'un niveau d'organisation à un autre, chaque modèle d'exploitation a été pris comme chorème élémentaire pour participer à la compréhension et à l'exploration du niveau territorial (Lardon, 2006a). Aux deux niveaux, le choix d'un nombre réduit de chorèmes rend compte par combinaison de la diversité des situations observables. Les dynamiques spatiales observées à l'échelle du territoire, ont permis de re-questionner les modèles établis à l'échelle des exploitations. Plus que les produits finaux (modèles graphiques), c'est l'ensemble du processus d'élaboration qui présente un intérêt en termes d'analyse des processus de changement.

\section{LA RÉORGANISATION SPATIALE DES ACTIVITÉS AGRICOLES INTERROGE LA PERTINENCE DES ZONAGES DU DISPOSITIF DE CONSERVATION}

Nos résultats montrent une homogénéité des processus de réorganisation des activités agricoles au sein des sous-zones, identifiées comme des unités paysagères intermédiaires opérationnelles pour discuter de la pertinence du zonage du dispositif de conservation, établi selon des critères essentiellement écologiques et forestiers.

L'intensification des cultures en zone de savane, avec la disparition des boisements (pins et eucalyptus) risque de contribuer à un accroissement des problèmes d'érosion; la disparition des zones de pâturage remet également en cause le développement de l'élevage bovin, indispensable au système de production rizicole. Paradoxalement, les modalités de mise en œuvre de la GCF ont encouragé une pérennisation de l'occupation agricole en zone forestière ; cette imbrication d'espaces d'agriculture et de forêt se révèle peu favorable pour l'amélioration des conditions de vie des ménages paysans et son impact sur la biodiversité reste à étudier (Carrière-Buchenschutz, 2006). Les impacts inattendus de la GCF résultent du fait que les dynamiques agricoles et les facteurs d'aménagement du milieu n’ont pas été pris en compte dans leur ensemble lors de l'élaboration du contrat de gestion. Toutes les exploitations ne présentent pas les mêmes capacités à s'adapter aux contraintes de la conservation. Contrairement au présupposé d'une homogénéité des communautés rurales dans les approches de gestion communautaire des forêts (Agrawal et Gibson, 1999), des systèmes de production plus ou moins dépendants des ressources forestières coexistent au sein des territoires villageois. On observe déjà dans certains cas, une évolution vers des systèmes de production moins consommateurs d'espace, avec le développement de cultures de rente et de pratiques plus intensives. Mais les exploitations qui en ont les moyens, ne sont localisées que dans certaines zones du territoire (zones proches d'axes de communication ou des zones d'habitation) et correspondent à celles qui sont déjà les plus capitalisées et les moins sensibles aux contraintes de la conservation. Dans une logique de zonage, les plans d'aménagement devraient donc faire référence aussi bien à la gestion forestière qu'à l'occupation agricole, en différenciant les règles de gestion par sous-zones (en fonction, par exemple, des dynamiques d'aménagement des rizières et de la localisation des noyaux de peuplement), voire par type d'exploitation.

\section{LES MODÈLES PRODUITS COMME SUPPORTS DE DISCUSSION}

La modélisation graphique peut constituer une étape intermédiaire d'une démarche plus globale d'analyse du territoire et des enjeux liés à la gestion conjointe du développement agricole et de la conservation des forêts. Les représentations spatiales permettent de visualiser et d'expliquer les changements paysagers observés et de rendre compte de la logique propre des exploitations. 
AurÉlie TOILLIER

L'accompagnement des recompositions des différents types d'exploitations dans leur territoire pourrait aider à résoudre certains blocages rencontrés actuellement dans l'application des mesures de conservation. Dans cette perspective, cet outil doit devenir suffisamment explicite pour permettre un échange avec les acteurs autour de scenarii de gestion de l'espace rural. Il s'agit essentiellement d'intégrer les points de vue des paysans, via leurs propres représentations de l'espace et des changements. Des discussions autour de représentations partagées du territoire devraient renforcer leur degré d'implication (Lardon, 2006b) et leurs capacités de négociation autour du contrat de gestion des forêts. L'enjeu est de passer véritablement d'un aménagement sectoriel par et pour la conservation, à un développement local planifié qui prenne mieux en compte les besoins de populations qui vivent de l'agriculture, en compatibilité avec les objectifs de conservation.

\section{Bibliographie}

Agrawal (A.), Gibson (C.), 1999. - « Enchantment and disenchantment: the role of community in natural resource conservation », World Development, 27, n 4, p. 629-649.

Albaladejo (C.), LARDON (S.), 1990. - « La dynamique spatiale d'une zone pionnière agricole en Argentine », Mappemonde, $\mathrm{n}^{\circ}$ 4, p. 37-41.

Batterbury (S.), 1998. - « Local environmental management, land degradation and the "gestion des terroirs" approach in West Africa: Policies and pitfalls », Journal of International development, $\mathrm{n}^{\circ}$ 10, p. 871-898.

Benoît (M.), Deffontaines (J.-P.), Lardon (S.), 2006. - « Acteurs et territoires locaux. Vers une géoagronomie de l'aménagement », Éditions INRA, Savoir faire, 174 p.

Blanc-Pamard (C.), Milleville (P.), 1991. - «Pratiques paysannes, perception du milieu et systèmes agraires », dans Dynamique des systèmes agraires à travers champs agronomes et géographes, Paris, ORSTOM, p. 101-137.

Blanc-Pamard (C.) et Rakoto (H.), 2007. - « Normes environnementales, transferts de gestion et recompositions territoriales en pays betsileo (Madagascar). La gestion contractualisée des forêts », Natures Sciences Sociétés, n 15, p. 253-268.

Bonin (M.), 2002. - « Nouvelles fonctions de l'agriculture et dynamique des exploitations. Une analyse chorématique dans les monts d'Ardèche », Mappemonde, n 62, p. 11-16.

BONIN (M.), LARDON (S.), 2002 - « Territoire et recomposition des exploitations agricoles : diversification des activités et des pratiques de gestion de l'espace. Application au Parc Naturel Régional des Monts d'Ardèche et au Causse Méjean », Étude et Recherche sur les Systèmes Agraires et le Développement. $n^{\circ} 33$, p. 131-148.

Bonin (M.), Caron (P.), Cheylan (J.-P.), Clouet (Y.), Clouet (P.), Thinon (P.), 2001. - « Territoire, zonage et modélisation graphique : recherche-action et apprentissage », Géocarrefour, vol. 73, n 3, p. 241-252.

Brunet (R.), 1980. - « La composition des modèles dans l'analyse spatiale. », L'espace Géographique, n 4, p. $253-265$.

Brunet (R.), 1986. - « La carte modèle et les chorèmes », Mappemonde, n 4, p. 2-6.

CARRiÈre-Buchsenschutz (S.), 2006. - «L'urgence de la confirmation par la science. Du rôle écologique du corridor forestier de Fianarantsoa », Études rurales, n 178, p. 181-196.

DefFontaines (J.-P.), 1996. - « Enjeux spatiaux en agronomie », Comptes rendus de l'Académie d'Agriculture de France, vol. 82, n 8, p. 151-156.

Deffontaines (J.-P.), 2006. - «Analyse du paysage », dans Benoît M., DefFontaines J.-P., Lardon S., Acteurs et territoires locaux. Vers une géoagronomie de l'aménagement, Éditions INRA, Savoir faire, p. 69-84.

Froger (G.), Méral (P.), Herimandimby (V.), 2004. - « The expansion of participatory governance in the environmental policies of developing countries: the example of Madagascar », International Journal of Sustainable Development, vol. 7, $\mathrm{n}^{\circ}$ 2, p. 164-184.

GAFsi (M.), Dugué (P.), JAmin (J.-Y.), Broissier (J.) (dir.), 2007. - Exploitations agricoles familiales en Afrique de l'Ouest et du Centre, Éditions Quae, 472 p. 
Lardon (S.), DefFontaines (J.-P.), Osty (P.-L.), 2001. - « Pour une agronomie du territoire : prendre en compte l'espace pour accompagner le changement technique », Comptes rendus de l'Académie d'Agriculture de France, n ${ }^{\circ}$, p. 187-198.

Lardon (S.), Le Ber (F.), MetzGer (J.-L.), Osty (P.-L.), 2005. - « Une démarche et un outil pour modéliser des organisations spatiales agricoles et raisonner à partir de cas d'exploitations agricoles », Géomatique, vol. 15, n³, p. 263-280.

LARDON (S.), 2006a. - « Organisation spatiale des exploitations agricoles dans des territoires locaux », dans Benoît (M.), DefFontaines (J.-P.), LaRdon (S.) (dir.), Acteurs et territoires locaux. Vers une géoagronomie de l'aménagement, Éditions INRA, Savoir faire, p. 103-129.

LARDOn (S.), 2006b. - « La modélisation graphique », dans Benoît (M.), DefFontaines (J.-P.), Lardon (S.), Acteurs et territoires locanx. Vers une géoagronomie de l'aménagement, Éditions INRA, Savoir faire, p. 33-55.

Montagne (P.), RamamonjisoA (B.), 2006. - « Politiques forestières à Madagascar entre répression et autonomie des acteurs ", Économie Rurale, $n^{\circ} 294-295$, p. 9-26.

Montagne (P.), Razanamaharo (Z.), Cooke (A.) (dir.), 2008. - Tanteza, le transfert de gestion à Madagascar; dix ans d'efforts, Antananarivo, CITE, 207 p.

Moreau (S.), 2002. - Les gens de la lisière. La forêt, l'arbre et la construction paysanne Sud-Betsileo, Madagascar, Thèse de doctorat, Université de Paris X, 667 p.

Osty (P.-L.), Lardon (S.), De Sainte-Marie (C.), 1998. - « Comment analyser les transformations de l'activité productive des agriculteurs? Propositions à partir des systèmes techniques de production ", Étude et Recherche sur les Systèmes Agraires et le Développement, n 31, p. 397-413.

Randriamalala (J.), Serpantié (G.), Carrière (S.), 2007. - « Influence des pratiques agricoles et du milieu sur la diversité des jachères d'origine forestière (Haute-Terre, Madagascar) », Revue d'Écologie la Terre et la Vie, vol. 62, n² 2-3, p. 169-189.

Thinon (P.), Deffontaines (J.-P.), 1999. - « Partage de l'espace rural pour la gestion de problèmes environnementaux et paysagers dans le Vexin français », Cahiers Agriculture, n 4, p. 434-439.

Toillier (A.), Serpantié (G.), Hervé (D.), Lardon (S.), 2009. - « Livelihood strategies and land use changes in response to conservation: an insight into pitfalls of community-based forest management in Madagascar », Journal of Sustainable Forestry, volume 27, issue 3-4 (à paraître).

Cet article a été reçu le 28 avril 2008 et a été définitivement accepté le 19 novembre 2008 